\title{
Healthcare Resources Consumed by Foreign Patients in the Public Hospitals of East Macedonia and Thrace
}

\author{
Christos Tsitsakis ${ }^{1}$ Christos Batzios ${ }^{2}$, Giannoula Florou ${ }^{3}$, Anastasios \\ Karasavvoglou $^{4}$, Persefoni Polychronidou ${ }^{5}$
}

\begin{abstract}
:
The rising healthcare expenditures as percentage of GDP is a reality that all developed countries have to deal with. Epidemiologic standards have changed dramatically since the past. The rapid development of knowledge in the scientific field of medicine and the new state of the art medical technology has led to new treatments for various health problems. Prior fatal diseases have become chronic, and life expectancy has risen significantly, leading healthcare costs to explosion. Healthcare spending in Greece has been increased between 2000 and 2009 at a rapid rate of $6.1 \%$ per year, but it has dropped the following years, driven by a sharp reduction in public spending on health care as part of government-wide efforts to reduce the large budgetary deficit. This situation has caused an increasing offence sense in population. On the other hand, the last years, Greece has become an immigrant (legal and illegal) reception centre.This study presents comparative data about the consumed resources by Greek and foreign patients in the public hospitals of east Macedonia and Thrace for the years 2006-2010.
\end{abstract}

Key Words: Healthcare Resources, Public Hospitals, Migrants

JEL Classification: D23, D74

\footnotetext{
${ }^{1}$ Technological Educational Institute of Central Greece, 34400 Psaxna Euboea, Greece, e mail: tsitsakis@teihal.gr

${ }^{2}$ Lab. of Animal Production Economics, School of Veterinary Medicine, Faculty of Health Sciences, Aristotle University of Thessaloniki, 54124 Thessaloniki, GREECE, batzios@ vet.auth.gr

${ }_{3}^{3}$ Department of Accountancy and Finance, Eastern Macedonia and Thrace Institute of Technology, Agios Loukas, 65404 Kavala, GREECE, gflorou@teikav.edu.gr

${ }^{4}$ Department of Accountancy and Finance, Eastern Macedonia and Thrace Institute of Technology, Agios Loukas, 65404 Kavala, GREECE, akarasa@ teikav.edu.gr

${ }^{5}$ Department of Accountancy and Finance, Eastern Macedonia and Thrace Institute of Technology, Agios Loukas, 65404 Kavala, GREECE, polychr@ teikav.edu.gr
} 


\section{Introduction}

The Greek National Health System (ESY- Ethniko Systima Ygeias) was established in 1983 and provides health care for all residents of Greece. Primary health care services are provided mainly through the social insurance institutions' specialists, the rural health centres and the outpatient departments of regional and district hospitals. Secondary and tertiary care is provided by ESY public hospitals and private forprofit hospitals.

There are 132 public hospitals under the ESY out of which 84 are general hospitals, 7 are university hospitals, 23 are specialized hospitals and 18 are small hospitals/health centres. Most public hospitals have a capacity of 100-200 beds and offer mainly secondary health care, while 32 of them have a capacity of more than 400 beds and offer tertiary health care, as they are equipped with advanced technology and are staffed with specialized personnel. In addition, there are 14 military hospitals funded by the Ministry of Defence, 5 hospitals funded exclusively through the IKA budget, 2 university hospitals which receive extra funds from the Ministry of Education and 2 hospitals under the supervision of the Ministry of Justice, serving the needs of prisoners (Economou 2010).

The health system in Greece is a mixed system, financed by a combination of taxbased and insurance-based statutory financing (Tragakes and Polyzos 1996). Greek public hospitals are funded partially from the State Budget and partially from the fees for the provided services. Fees are paid in most cases either by public social insurance organizations or by private insurance organizations. Social insurance is mandatory for all employees and includes health insurance. Registration with such organizations requires part or full time employment.

The public health system covers Greek, European Union and non European Union citizens according to the norms and standards of international and European social security law and bilateral social insurance conventions and agreements. If a foreigner lives in Greece and pays social security contributions, he has the same level of access to public healthcare services. Unemployed citizens are covered for a period up to 12 months. Poor citizens are characterized as needy provided they can prove their economic status and have free access to health centers and public hospitals. The uninsured have no free access to health services and must pay for them.

In 2010, the Greek economy started to face a deep structural crisis, the main features of which are a large fiscal deficit, huge public debt and the continuous erosion of the country's competitive position. According to OECD data, health spending in Greece increased at a rapid rate of $5.7 \%$ per year in real terms on average between 2000 and 2009, but fell by $11 \%$ in both 2010 and 2011 . These cuts were driven by a sharp 
reduction in public spending on health as part of government-wide efforts to reduce the large budgetary deficit.

Most of the reductions in public spending have been achieved through cuts in wages and actual reductions in the number of health workers, as well as price reductions for pharmaceuticals (OECD, 2012). From the beginning of the crisis, the Ministry of Health has considered a range of proposals for reform, all aiming to achieve greater efficiency and reduce expenditure, responding to one of the IMF's key loan conditions, namely that public health expenditures must not exceed 6\% of GDP (Kentikelenis and Papanicolas, 2012). The Minister of Health called for a $40 \%$ reduction in hospital budgets, but only a few hospitals achieved this target. On the other hand, the crisis caused an increased utilization of public health services. This reflects the inability to afford private sector health services, which previously played a large role in Greece (Kentikelenis et al., 2011).

This conflict between the demand for and offer of healthcare services is causing an increasing sense of isolation and injustice in the population which is sometimes expressed by the phenomena of social exclusion and persecution especially for immigrants, minorities and other vulnerable groups who become 'scapegoats' for populist politicians and the media. In the past, Greece was predominantly a country from which people emigrated. However, there has been a gradual reversal in the last three decades and Greece has recently emerged as a country which is attracting immigrants. Most of these come from neighboring countries mainly from Albania, but there are a considerable number of economic immigrants and asylum seekers who come from Eastern Europe, the former USSR, the Middle East and several Asian and African countries.

According to the National Statistical Service of Greece (ESYE), in a population of $11,192,849$ people in 2007 , there were 683,751 immigrants with valid residence permits. The same report has estimated that there were 280,000 illegal immigrants. However, the new ESYE data from the 2011 census show an increase in the immigrant population. In a total population of $10,815,197$, there were 911,929 immigrants, while there is no report on the number of illegal immigrants.

As mentioned above, legal insured immigrants have the same access to hospital services as Greek citizens. However, for the undocumented immigrants, legislation prohibits healthcare provision with the exception of emergency treatment for life threatening illnesses as well as healthcare provision to underage children (Article 84/N.3386/2005). Infectious diseases, such as HIV, and childbirth, are normally considered as emergencies (Médecins du Monde 2009). However, in practice undocumented migrants often receive primary healthcare at rural health centers as well as from out-patient hospital services. Occasionally, secondary care might also be provided, albeit unofficially. 
The research was supported by the Project "Immigrants and Health Services - The case of Eastern Macedonia and Thrace region" that is co-funded by the European Union (European Social Fund) and National Resources - ARCHIMEDES III.

\section{The Study}

The region of Eastern Macedonia and Thrace consists of five prefectures: Drama, Evros, Kavala, Xanthi and Rodopi. The total population of the region according to the data of a 2011 census is 608,182 people. While data is available for the total number of immigrants in Greece $(911,929)$, no published information could be found on the number of immigrants in the Eastern Macedonia and Thrace region. The demand for healthcare services in the region is satisfied by six public hospitals described in Table 1:

Table 1: Public Hospitals of Eastern Macedonia and Thrace Region

\begin{tabular}{|l|l|l|l|}
\hline Hospital & Prefecture & City & $\begin{array}{l}\text { Capacity in } \\
\text { beds }\end{array}$ \\
\hline $\begin{array}{l}\text { General University Hospital of } \\
\text { Alexandroupolis }\end{array}$ & Evros & Alexandroupolis & 500 \\
\hline General Hospital of Didimoticho & Evros & Didimoticho & 112 \\
\hline $\begin{array}{l}\text { General Hospital of Komotini } \\
\text { "Sismanoglio" }\end{array}$ & Rodopi & Komotini & 238 \\
\hline General Hospital of Kavala & Kavala & Kavala & 400 \\
\hline General Hospital of Xanthi & Xanthi & Xanthi & 222 \\
\hline General Hospital of Drama & Drama & Drama & 270 \\
\hline
\end{tabular}

We collected data for only 5 out the six hospitals. Unfortunately, senior management at the University Hospital of Alexandroupolis refused to provide relevant data.

Collected data differ among hospitals due to the different practices in registry recording as well as the differences in the software that hospitals use. Collected data from the hospitals of Kavala, Xanthi and Didimoticho relate to the years 2005-2011, while the data from the hospitals of Drama and Komotini relate to the years 20082011.

Data include:

(a) the health insurance organization that covers the fees,

(b) the admission diagnosis,

(c) the ward of admission (for the hospitals of Kavala, Xanthi and Didimoticho)

(d) the length of stay,

(e) the ethnicity and

(f) the reimbursement amount. 
In order to proceed with our analysis, we recoded the insurance organization data, which diversified greatly in terms of how they were recorded, especially in the cases of uninsured patients. In some hospitals there were records labeled "needy" and other records labeled "undocumented", while in other hospitals there were records labeled "pending". In an initial recoding, we merged these cases into two major categories: "Uninsured" and "Needy". We kept the label "needy" as it was recorded in any relevant case, because it describes a person legally living in the country, who has been unemployed for over 12 months and has zero income. Such people have free access to healthcare services. In a second recoding, and in order to define the loss of the hospitals from uncollected receivables we merged all these cases under the label "uninsured", since all have the same loss impact in the hospitals' revenue.

In our initial recoding, we also kept the label "European health insurance card" for foreign nationals who owned such a card and were hospitalized free of charge as an interesting indication, while the other cases describing the different social security funds (IKA, OGA etc.) were merged and named "public insurance". In a second recoding we included the cases labeled "European Health Insurance Card" in the category "Public insurance", since it has the same positive impact on the collection of the amounts receivable from the hospitals. The remaining cases, concerning payments either directly from the patient or from a private insurance organization, initially remained as they were, distinguishing between different cases of people who are wealthy enough to be covered by a private insurance organization and people who did not have any kind of insurance (like the unemployed and/or undocumented) and were obliged to pay for the received healthcare services, either from their savings or by loaning the amount. In a second stage recoding, we merged these two cases and labeled them as "private insurance" since they have the same positive impact on the hospitals' revenue.

The data concerning the ethnicity of patients initially remained unchanged in order to map the countries of origin of the foreign patients. In a second stage analysis, they were recoded, and the cases, which numbered under 100, were merged into "Other European Union countries" and "Other Non European Union countries". In the final stage, all cases were recoded into three major categories: "Greece", "European Union countries" and "Non European Union countries". However, we have to mention that there is no indication whether the foreign patients are immigrants who live in the region or tourists who needed healthcare services during their holidays or foreigners from neighboring countries who trust the Greek doctors and hospitals and came to Greece to be treated.

The data concerning the length of stay is directly related to the data concerning the reimbursement amount since the reimbursement of Greek public hospitals is based on a standard per diem fee. 
The data concerning the admission diagnosis was ignored, since it was characterized by a wide diversification in the terms describing the ailment even for the same diseases and even in the same hospital. For example, in the hospital of Kavala in the case of patients with multiple sclerosis, there were records labeled "multiple sklerosis", "sklirinsi kata plakas" (the Greek term for the disease) or the abbreviation "MS". Moreover, the admission diagnosis is the initial "working" diagnosis documented by the patient's admitting or attending physician who determines whether inpatient care is necessary. In many cases, the definitive final diagnosis which is determined through studies, procedures, and consultations during the inpatient hospital stay, differs from the diagnosis on admission. So, cases can be found where the admission diagnosis was quite general (e.g. "lower back pain" or "belly pain") resulting in a three-day hospital stay. It is obvious that the belly pain was only a symptom not the disease. It would be quite interesting if we had the final diagnosis. It could be possible to structure a kind of epidemiologic profile of foreign inpatients, by the country of origin.

The data concerning the ward of admission was utilized to give an indication of the kind of health problems which foreign patients face. Table 2 below is a frequency table, mapping the countries of origin of foreign patients.

Table 2. Frequencies of Countries the Foreign Patients Come From

\begin{tabular}{|l|l|l|l|}
\hline Country & Frequency & Percent & $\begin{array}{l}\text { Cumulative } \\
\text { Percent }\end{array}$ \\
\hline Albania & 6,458 & $46.02 \%$ & $46.02 \%$ \\
\hline Bulgaria & 1,790 & $12.75 \%$ & $58.77 \%$ \\
\hline Georgia & 1,214 & $8.65 \%$ & $67.42 \%$ \\
\hline Russia & 1,145 & $8.16 \%$ & $75.58 \%$ \\
\hline Germany & 542 & $3.86 \%$ & $79.44 \%$ \\
\hline Armenia & 528 & $3.76 \%$ & $83.21 \%$ \\
\hline Moldavia & 210 & $1.50 \%$ & $84.70 \%$ \\
\hline $\begin{array}{l}\text { United } \\
\text { Kingdom }\end{array}$ & 183 & $1.30 \%$ & $86.01 \%$ \\
\hline Ukraine & 160 & $1.14 \%$ & $87.15 \%$ \\
\hline Turkey & 146 & $1.04 \%$ & $88.19 \%$ \\
\hline Kazakhstan & 129 & $0.92 \%$ & $89.11 \%$ \\
\hline Serbia & 124 & $0.88 \%$ & $89.99 \%$ \\
\hline Egypt & 118 & $0.84 \%$ & $90.83 \%$ \\
\hline Iraq & 117 & $0.83 \%$ & $91.66 \%$ \\
\hline Romania & 114 & $0.81 \%$ & $92.48 \%$ \\
\hline
\end{tabular}


Healthcare Resources Consumed by Foreign Patients in the Public Hospitals of east Macedonia and Thrace

\begin{tabular}{|l|l|l|l|}
\hline Italy & 77 & $0.55 \%$ & $93.02 \%$ \\
\hline Syria & 64 & $0.46 \%$ & $93.48 \%$ \\
\hline Poland & 58 & $0.41 \%$ & $93.89 \%$ \\
\hline Uzbekistan & 55 & $0.39 \%$ & $94.29 \%$ \\
\hline China & 51 & $0.36 \%$ & $94.65 \%$ \\
\hline Cyprus & 47 & $0.33 \%$ & $94.98 \%$ \\
\hline Netherlands & 45 & $0.32 \%$ & $95.30 \%$ \\
\hline Pakistan & 43 & $0.31 \%$ & $95.61 \%$ \\
\hline Argentine & 42 & $0.30 \%$ & $95.91 \%$ \\
\hline FYROM & 34 & $0.24 \%$ & $96.15 \%$ \\
\hline Czech & 32 & $0.23 \%$ & $96.38 \%$ \\
\hline Republic & & & \\
\hline Country & Frequency & Percent & Cumulative \\
\hline Palestine & 32 & $0.23 \%$ & $96.61 \%$ \\
\hline Spain & 30 & $0.21 \%$ & $96.82 \%$ \\
\hline France & 28 & $0.20 \%$ & $97.02 \%$ \\
\hline Afghanistan & 27 & $0.19 \%$ & $97.21 \%$ \\
\hline Sweden & 26 & $0.19 \%$ & $97.40 \%$ \\
\hline Slovakia & 23 & $0.16 \%$ & $97.56 \%$ \\
\hline Somalia & 23 & $0.16 \%$ & $97.73 \%$ \\
\hline Belarus & 22 & $0.16 \%$ & $97.88 \%$ \\
\hline Austria & 21 & $0.15 \%$ & $98.03 \%$ \\
\hline Denmark & 21 & $0.15 \%$ & $98.18 \%$ \\
\hline Chile & 20 & $0.14 \%$ & $98.33 \%$ \\
\hline Hungary & 20 & $0.14 \%$ & $98.47 \%$ \\
\hline Iran & 20 & $0.14 \%$ & $98.61 \%$ \\
\hline USA & 19 & $0.14 \%$ & $98.75 \%$ \\
\hline Switzerland & 18 & $0.13 \%$ & $98.87 \%$ \\
\hline Croatia & 14 & $0.10 \%$ & $98.97 \%$ \\
\hline India & 14 & $0.10 \%$ & $99.07 \%$ \\
\hline Belgium & 11 & $0.08 \%$ & $99.15 \%$ \\
\hline Brazil & 10 & $0.07 \%$ & $99.22 \%$ \\
\hline Australia & 8 & $0.06 \%$ & $99.28 \%$ \\
\hline Jordan & 8 & $0.06 \%$ & $99.34 \%$ \\
\hline & & & \\
\hline
\end{tabular}


International Journal of Economics \& Business Administration, I(2)2014

126 C. Tsitsakis - C. Batzios - G. Florou - A. Karasavvoglou - P. Polychronidou

\begin{tabular}{|l|l|l|l|}
\hline Portugal & 8 & $0.06 \%$ & $99.39 \%$ \\
\hline Algeria & 7 & $0.05 \%$ & $99.44 \%$ \\
\hline Lithuania & 6 & $0.04 \%$ & $99.49 \%$ \\
\hline Morocco & 6 & $0.04 \%$ & $99.53 \%$ \\
\hline Canada & 5 & $0.04 \%$ & $99.57 \%$ \\
\hline Nigeria & 5 & $0.04 \%$ & $99.60 \%$ \\
\hline Angola & 4 & $0.03 \%$ & $99.63 \%$ \\
\hline Eritrea & 4 & $0.03 \%$ & $99.66 \%$ \\
\hline Norway & 4 & $0.03 \%$ & $99.69 \%$ \\
\hline Uknown & 4 & $0.03 \%$ & $99.71 \%$ \\
\hline Bangladesh & 3 & $0.02 \%$ & $99.74 \%$ \\
\hline Dominikus & 3 & $0.02 \%$ & $99.76 \%$ \\
\hline Japan & 3 & $0.02 \%$ & $99.78 \%$ \\
\hline Myanmar & 3 & $0.02 \%$ & $99.80 \%$ \\
\hline Philippines & 3 & $0.02 \%$ & $99.82 \%$ \\
\hline Tanzania & 3 & $0.02 \%$ & $99.84 \%$ \\
\hline Azerbaijan & 2 & $0.01 \%$ & $99.86 \%$ \\
\hline Bosnia & 2 & $0.01 \%$ & $99.87 \%$ \\
\hline Slovenia & 2 & $0.01 \%$ & $99.89 \%$ \\
\hline Srilanka & 2 & $0.01 \%$ & $99.90 \%$ \\
\hline Tajikistan & 2 & $0.01 \%$ & $99.91 \%$ \\
\hline Kashmir & 1 & $0.01 \%$ & $99.92 \%$ \\
\hline Kenya & 1 & $0.01 \%$ & $99.93 \%$ \\
\hline Korea & 1 & $0.01 \%$ & $99.94 \%$ \\
\hline Country & Frequency & Percent & $\begin{array}{l}\text { Cumulative } \\
\text { Percent }\end{array}$ \\
\hline Lebanon & 1 & $0.01 \%$ & $99.94 \%$ \\
\hline Mauritania & 1 & $0.01 \%$ & $99.95 \%$ \\
\hline Mongolia & 1 & $0.01 \%$ & $99.96 \%$ \\
\hline New Zealand & 1 & $0.01 \%$ & $99.96 \%$ \\
\hline Ruanda & 1 & $0.01 \%$ & $99.97 \%$ \\
\hline Sudan & 1 & $0.01 \%$ & $99.98 \%$ \\
\hline Tunis & 1 & $0.01 \%$ & $99.99 \%$ \\
\hline Turkmenistan & 1 & $0.01 \%$ & $99.99 \%$ \\
\hline Venezouela & 1 & $0.01 \%$ & $100.00 \%$ \\
\hline & & & \\
\hline
\end{tabular}




\begin{tabular}{|l|l|l|l|}
\hline Total & 14,034 & $100 \%$ & \\
\hline
\end{tabular}

We realize that about half of foreign patients come from Albania, which is the source country for the bulk of immigrants to Greece. In the 90s, Greece became a popular haven for Albanians (Vidali 1999), and they still constitute the largest immigrant group in the country. Today, most of them are incorporated into the Greek society and there is already a second, born in Greece, generation.

Bulgarians, Georgians and Russians follow, with percentages totalling $12.75 \%$, $8.65 \%$ and $8.16 \%$ respectively. Traditionally, Greece was an attractive destination for these countries, because of some features such as:

- Large informal economies (20-30\%).

- Large agricultural sectors and other labour-intensive economic sectors.

- Increasing elderly populations in need of welfare not provided by the state.

These features tend to favour the illegal and semi-legal employment of immigrants, such as seasonal farm labourers, housekeepers and construction workers (BaldwinEdwards, 2004). The cultural and religious similarities (Greeks are Orthodox Christians as are Bulgarians, Georgians and Russians) seem to be another factor which affects the preference of immigrants from these countries (Maroufof, 2013). The following Table 3 shows the geographic distribution of these groups among the hospitals in the region of Eastern Macedonia and Thrace.

Table 3: Foreign Patients by Nationality and Hospital in the Region of Eastern Macedonia and Thrace for the years 2005-20111

\begin{tabular}{|c|c|c|c|c|c|c|c|}
\hline & \multicolumn{5}{|l|}{ Hospital } & \multirow[b]{2}{*}{ Total } \\
\hline & & Didimoticho & Drama & Kavala & Komotini & Xanthi & \\
\hline \multirow[t]{4}{*}{ Albania } & Count & 12 & 259 & 5619 & 64 & 504 & 6,458 \\
\hline & $\begin{array}{c}\% \text { within } \\
\text { Nationality }\end{array}$ & $.2 \%$ & $4.0 \%$ & $87.0 \%$ & $1.0 \%$ & $7.8 \%$ & $100.0 \%$ \\
\hline & $\begin{array}{l}\% \text { within } \\
\text { Hospital }\end{array}$ & $1.7 \%$ & $43.3 \%$ & $54.8 \%$ & $6.5 \%$ & $33.9 \%$ & $46.0 \%$ \\
\hline & $\%$ of Total & $.1 \%$ & $1.8 \%$ & $40.0 \%$ & $.5 \%$ & $3.6 \%$ & $46.0 \%$ \\
\hline \multirow[t]{4}{*}{ Armenia } & Count & 82 & 4 & 183 & 66 & 193 & 528 \\
\hline & $\begin{array}{c}\% \text { within } \\
\text { Nationality }\end{array}$ & $15.5 \%$ & $.8 \%$ & $34.7 \%$ & $12.5 \%$ & $36.6 \%$ & $100.0 \%$ \\
\hline & $\begin{array}{l}\text { \% within } \\
\text { Hospital }\end{array}$ & $11.5 \%$ & $.7 \%$ & $1.8 \%$ & $6.7 \%$ & $13.0 \%$ & $3.8 \%$ \\
\hline & $\%$ of Total & $.6 \%$ & $.0 \%$ & $1.3 \%$ & $.5 \%$ & $1.4 \%$ & $3.8 \%$ \\
\hline
\end{tabular}


International Journal of Economics \& Business Administration, I(2)2014

128 C. Tsitsakis - C. Batzios - G. Florou - A. Karasavvoglou - P. Polychronidou

\begin{tabular}{|c|c|c|c|c|c|c|c|}
\hline Bulgaria & Count & 242 & 0 & 1158 & 103 & 287 & 1,790 \\
\cline { 2 - 7 } & $\begin{array}{c}\text { \% within } \\
\text { Nationality }\end{array}$ & $13.5 \%$ & $0.0 \%$ & $64.7 \%$ & $5.8 \%$ & $16.0 \%$ & $100.0 \%$ \\
\cline { 2 - 7 } & $\begin{array}{c}\% \text { within } \\
\text { Hospital }\end{array}$ & $34.0 \%$ & $0.0 \%$ & $11.3 \%$ & $10.4 \%$ & $19.3 \%$ & $12.8 \%$ \\
\cline { 2 - 7 } & $\%$ of Total & $1.7 \%$ & $0.0 \%$ & $8.3 \%$ & $.7 \%$ & $2.0 \%$ & $12.8 \%$ \\
\hline
\end{tabular}

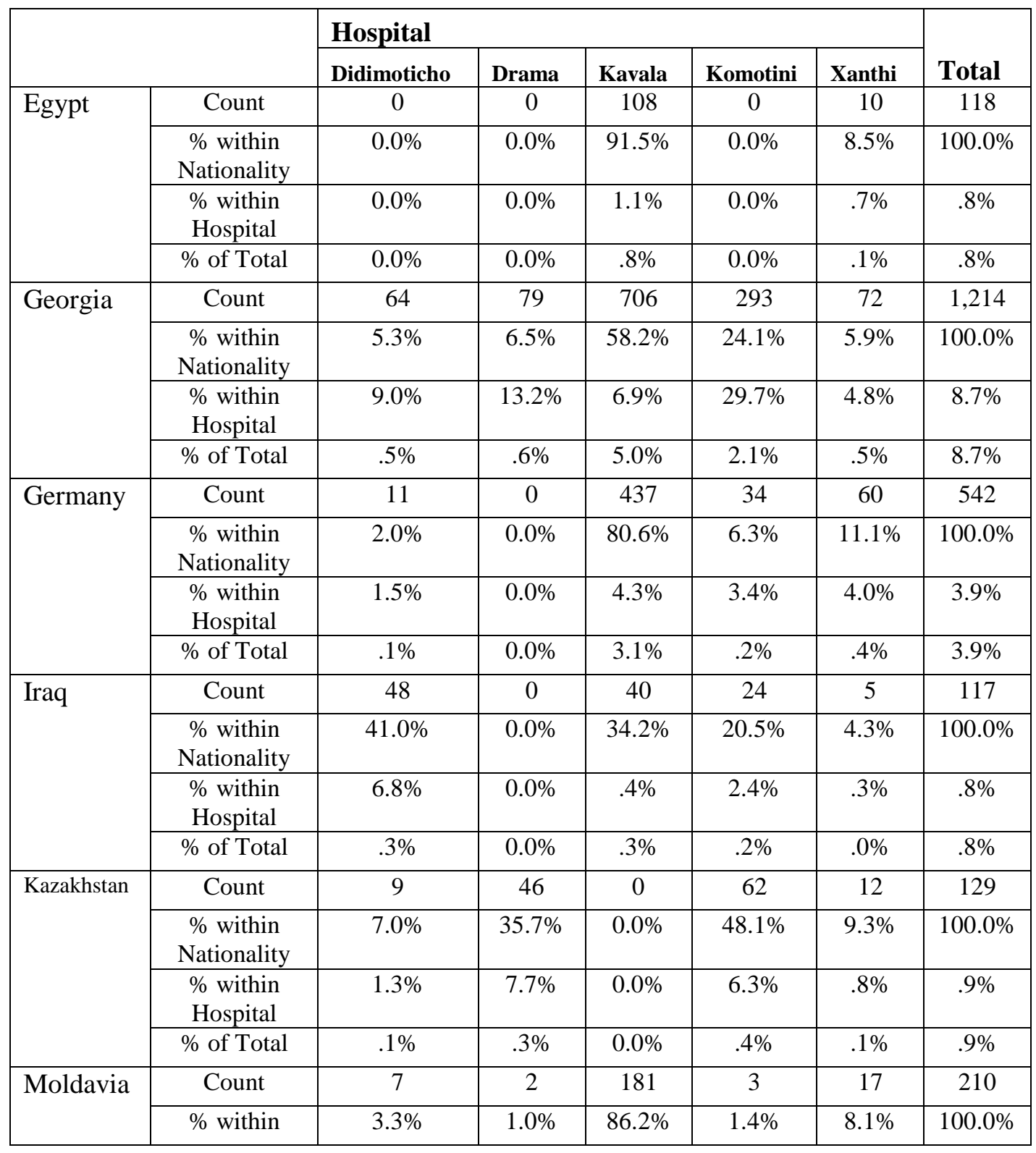


Healthcare Resources Consumed by Foreign Patients in the Public Hospitals of east Macedonia and Thrace

\begin{tabular}{|c|c|c|c|c|c|c|c|}
\hline & Nationality & & & & & & \\
\hline & $\begin{array}{l}\% \text { within } \\
\text { Hospital }\end{array}$ & $1.0 \%$ & $.3 \%$ & $1.8 \%$ & $.3 \%$ & $1.1 \%$ & $1.5 \%$ \\
\hline & $\%$ of Total & $.0 \%$ & $.0 \%$ & $1.3 \%$ & $.0 \%$ & $.1 \%$ & $1.5 \%$ \\
\hline \multirow[t]{4}{*}{ Romania } & Count & 20 & 0 & 43 & 17 & 34 & 114 \\
\hline & $\begin{array}{c}\% \text { within } \\
\text { Nationality }\end{array}$ & $17.5 \%$ & $0.0 \%$ & $37.7 \%$ & $14.9 \%$ & $29.8 \%$ & $100.0 \%$ \\
\hline & $\begin{array}{l}\% \text { within } \\
\text { Hospital }\end{array}$ & $2.8 \%$ & $0.0 \%$ & $.4 \%$ & $1.7 \%$ & $2.3 \%$ & $.8 \%$ \\
\hline & $\%$ of Total & $.1 \%$ & $0.0 \%$ & $.3 \%$ & $.1 \%$ & $.2 \%$ & $.8 \%$ \\
\hline \multirow[t]{4}{*}{ Russia } & Count & 29 & 162 & 731 & 152 & 71 & 1,145 \\
\hline & $\begin{array}{c}\% \text { within } \\
\text { Nationality }\end{array}$ & $2.5 \%$ & $14.1 \%$ & $63.8 \%$ & $13.3 \%$ & $6.2 \%$ & $100.0 \%$ \\
\hline & $\begin{array}{l}\% \text { within } \\
\text { Hospital }\end{array}$ & $4.1 \%$ & $27.1 \%$ & $7.1 \%$ & $15.4 \%$ & $4.8 \%$ & $8.2 \%$ \\
\hline & $\%$ of Total & $.2 \%$ & $1.2 \%$ & $5.2 \%$ & $1.1 \%$ & $.5 \%$ & $8.2 \%$ \\
\hline \multirow[t]{4}{*}{ Serbia } & Count & 0 & 0 & 113 & 0 & 11 & 124 \\
\hline & $\begin{array}{c}\% \text { within } \\
\text { Nationality }\end{array}$ & $0.0 \%$ & $0.0 \%$ & $91.1 \%$ & $0.0 \%$ & $8.9 \%$ & $100.0 \%$ \\
\hline & $\begin{array}{l}\% \text { within } \\
\text { Hospital }\end{array}$ & $0.0 \%$ & $0.0 \%$ & $1.1 \%$ & $0.0 \%$ & $.7 \%$ & $.9 \%$ \\
\hline & $\%$ of Total & $0.0 \%$ & $0.0 \%$ & $.8 \%$ & $0.0 \%$ & $.1 \%$ & $.9 \%$ \\
\hline \multirow[t]{4}{*}{ Turkey } & Count & 24 & 5 & 32 & 51 & 34 & 146 \\
\hline & $\begin{array}{c}\% \text { within } \\
\text { Nationality }\end{array}$ & $16.4 \%$ & $3.4 \%$ & $21.9 \%$ & $34.9 \%$ & $23.3 \%$ & $100.0 \%$ \\
\hline & $\begin{array}{l}\% \text { within } \\
\text { Hospital }\end{array}$ & $3.4 \%$ & $.8 \%$ & $.3 \%$ & $5.2 \%$ & $2.3 \%$ & $1.0 \%$ \\
\hline & $\%$ of Total & $.2 \%$ & $.0 \%$ & $.2 \%$ & $.4 \%$ & $.2 \%$ & $1.0 \%$ \\
\hline
\end{tabular}

\begin{tabular}{|c|c|c|c|c|c|c|c|}
\hline & & \multicolumn{5}{|l|}{ Hospital } & \multirow[b]{2}{*}{ Total } \\
\hline & & Didimoticho & Drama & Kavala & Komotini & Xanthi & \\
\hline \multirow[t]{4}{*}{ Ukraine } & Count & 4 & 7 & 112 & 12 & 25 & 160 \\
\hline & $\begin{array}{c}\% \text { within } \\
\text { Nationality }\end{array}$ & $2.5 \%$ & $4.4 \%$ & $70.0 \%$ & $7.5 \%$ & $15.6 \%$ & $100.0 \%$ \\
\hline & $\begin{array}{c}\% \text { within } \\
\text { Hospital }\end{array}$ & $.6 \%$ & $1.2 \%$ & $1.1 \%$ & $1.2 \%$ & $1.7 \%$ & $1.1 \%$ \\
\hline & $\%$ of Total & $.0 \%$ & $.0 \%$ & $.8 \%$ & $.1 \%$ & $.2 \%$ & $1.1 \%$ \\
\hline \multirow{3}{*}{$\begin{array}{l}\text { United } \\
\text { Kingdom }\end{array}$} & Count & 0 & 0 & 163 & 4 & 16 & 183 \\
\hline & $\begin{array}{c}\% \text { within } \\
\text { Nationality }\end{array}$ & $0.0 \%$ & $0.0 \%$ & $89.1 \%$ & $2.2 \%$ & $8.7 \%$ & $100.0 \%$ \\
\hline & $\%$ within & $0.0 \%$ & $0.0 \%$ & $1.6 \%$ & $.4 \%$ & $1.1 \%$ & $1.3 \%$ \\
\hline
\end{tabular}


International Journal of Economics \& Business Administration, I(2)2014

130 C. Tsitsakis - C. Batzios - G. Florou - A. Karasavvoglou - P. Polychronidou

\begin{tabular}{|l|c|c|c|c|c|c|c|}
\hline & Hospital & & & & & & \\
\cline { 2 - 8 } & $\%$ of Total & $0.0 \%$ & $0.0 \%$ & $1.2 \%$ & $.0 \%$ & $.1 \%$ & $1.3 \%$ \\
\hline \multirow{4}{*}{$\begin{array}{l}\text { Other EU } \\
\text { Countries }\end{array}$} & Count & 26 & 0 & 370 & 23 & 36 & 455 \\
\cline { 2 - 8 } & $\begin{array}{c}\% \text { within } \\
\text { Nationality }\end{array}$ & $5.7 \%$ & $0.0 \%$ & $81.3 \%$ & $5.1 \%$ & $7.9 \%$ & $100.0 \%$ \\
\cline { 2 - 8 } & $\begin{array}{c}\% \text { within } \\
\text { Hospital }\end{array}$ & $3.7 \%$ & $0.0 \%$ & $3.6 \%$ & $2.3 \%$ & $2.4 \%$ & $3.2 \%$ \\
\cline { 2 - 8 } & $\%$ of Total & $.2 \%$ & $0.0 \%$ & $2.6 \%$ & $.2 \%$ & $.3 \%$ & $3.2 \%$ \\
\hline \multirow{4}{*}{\begin{tabular}{l} 
Other $\begin{array}{l}\text { Non EU } \\
\text { Countries }\end{array}$ \\
\cline { 2 - 8 }
\end{tabular}} & $\begin{array}{c}\text { Count } \\
\text { Nationality }\end{array}$ & 133 & 34 & 253 & 80 & 101 & 601 \\
\cline { 2 - 8 } & $\begin{array}{c}\% \text { within } \\
\text { Hospital }\end{array}$ & $18.7 \%$ & $5.7 \%$ & $2.5 \%$ & $8.1 \%$ & $6.8 \%$ & $4.3 \%$ \\
\cline { 2 - 8 } & $\%$ of Total & $.9 \%$ & $.2 \%$ & $1.8 \%$ & $.6 \%$ & $.7 \%$ & $4.3 \%$ \\
\hline \multirow{4}{*}{ Total } & Count & 711 & 598 & 10,249 & 988 & 1.488 & 14,034 \\
\cline { 2 - 8 } & $\begin{array}{c}\% \text { within } \\
\text { Nationality }\end{array}$ & $5.1 \%$ & $4.3 \%$ & $73.0 \%$ & $7.0 \%$ & $10.6 \%$ & $100.0 \%$ \\
\cline { 2 - 8 } & $\begin{array}{c}\% \text { within } \\
\text { Hospital }\end{array}$ & $100.0 \%$ & $100.0 \%$ & $100.0 \%$ & $100.0 \%$ & $100.0 \%$ & $100.0 \%$ \\
\cline { 2 - 8 } & $\%$ of Total & $5.1 \%$ & $4.3 \%$ & $73.0 \%$ & $7.0 \%$ & $10.6 \%$ & $100.0 \%$ \\
\hline
\end{tabular}

As we can see from the data in table 3 . the bulk of foreign patients (73\%) of most nationalities were hospitalized in the hospital of Kavala. Exceptions are the patients from Armenia. Iraq. Kazakhstan and Turkey. for whom the highest percentages were hospitalized in the hospital of Xanthi. Didimoticho. Komotini and Komotini respectively. 
In the next table (4) we can see the kind of insurance by nationality.

Table 4: Foreign Patients by Nationality and Insurance in the Region of Eastern Macedonia and Thrace for the years 2005-2011

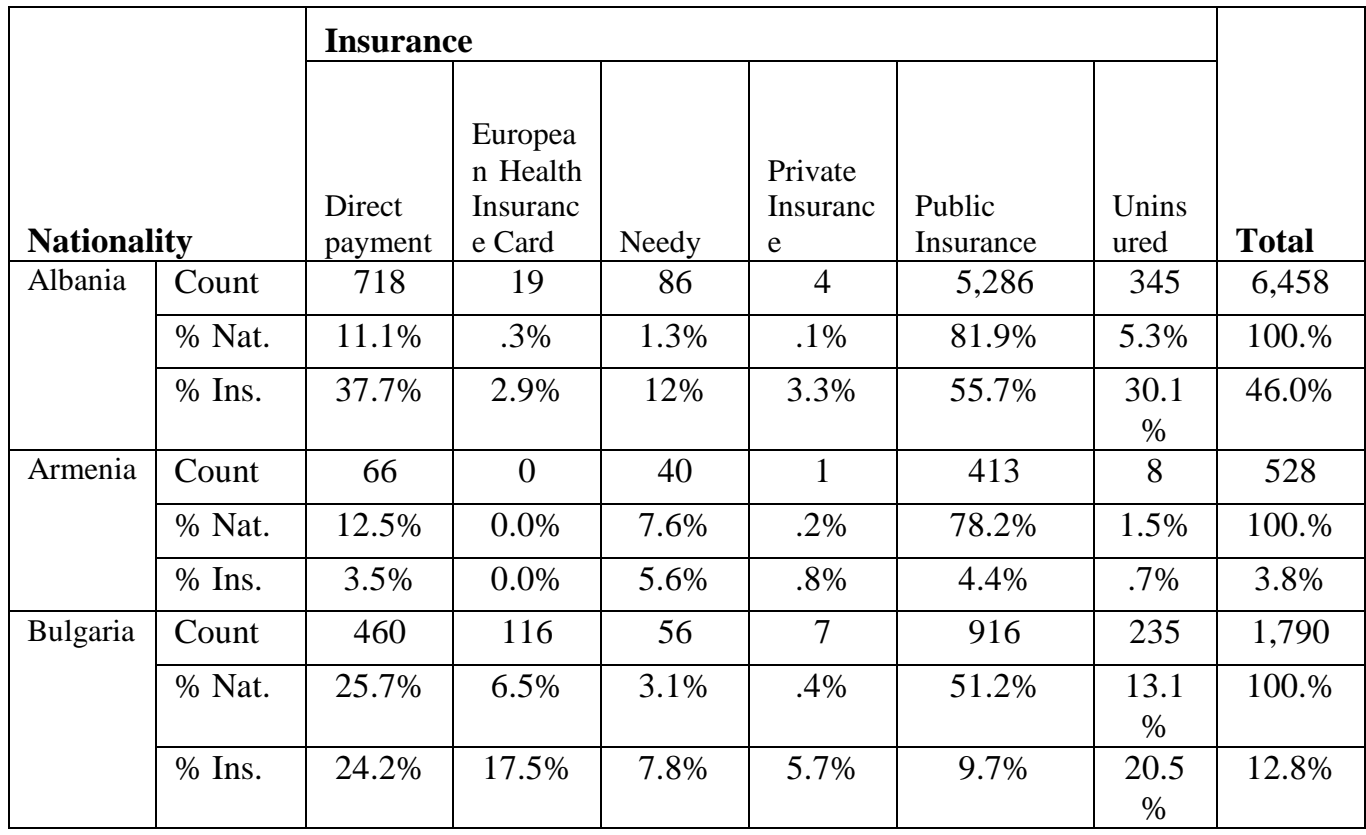

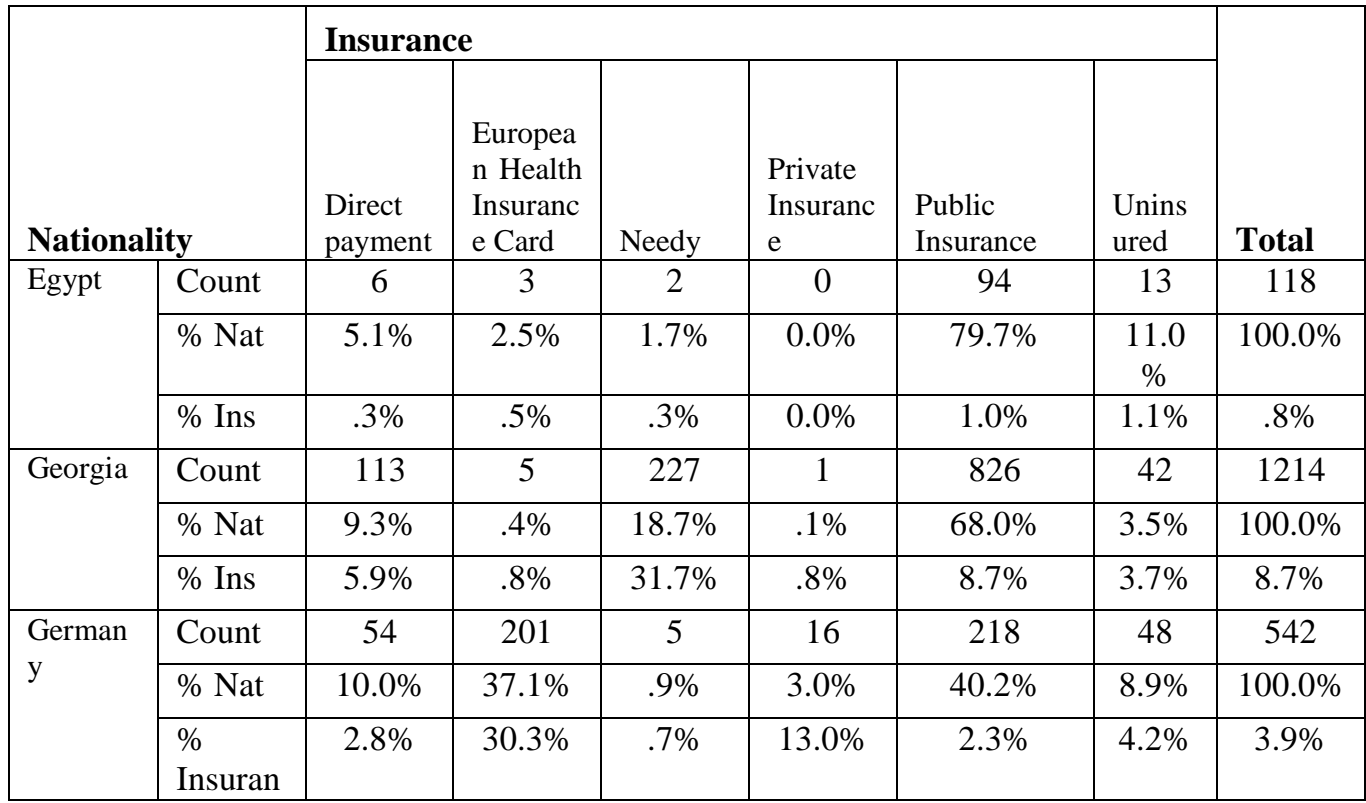


International Journal of Economics \& Business Administration, I(2)2014

132 C. Tsitsakis - C. Batzios - G. Florou - A. Karasavvoglou - P. Polychronidou

\begin{tabular}{|c|c|c|c|c|c|c|c|c|}
\hline & ce & & & & & & & \\
\hline \multirow[t]{3}{*}{ Iraq } & Count & 1 & 20 & 0 & 0 & 9 & 87 & 117 \\
\hline & $\begin{array}{l}\% \\
\text { Nationa } \\
\text { lity }\end{array}$ & $.9 \%$ & $17.1 \%$ & $0.0 \%$ & $0.0 \%$ & $7.7 \%$ & $\begin{array}{c}74.4 \\
\%\end{array}$ & $100.0 \%$ \\
\hline & $\begin{array}{l}\% \\
\text { Insuran } \\
\text { ce }\end{array}$ & $.1 \%$ & $3.0 \%$ & $0.0 \%$ & $0.0 \%$ & $.1 \%$ & $7.6 \%$ & $.8 \%$ \\
\hline \multirow{3}{*}{$\begin{array}{l}\text { Kazakhs } \\
\text { tan }\end{array}$} & Count & 15 & 0 & 19 & 0 & 95 & 0 & 129 \\
\hline & $\begin{array}{l}\% \\
\text { Nationa } \\
\text { lity }\end{array}$ & $11.6 \%$ & $0.0 \%$ & $14.7 \%$ & $0.0 \%$ & $73.6 \%$ & $0.0 \%$ & $100.0 \%$ \\
\hline & $\begin{array}{l}\% \\
\text { Insuran } \\
\text { ce }\end{array}$ & $.8 \%$ & $0.0 \%$ & $2.6 \%$ & $0.0 \%$ & $1.0 \%$ & $0.0 \%$ & $.9 \%$ \\
\hline \multirow{3}{*}{$\begin{array}{l}\text { Moldavi } \\
\text { a }\end{array}$} & Count & 6 & 0 & 0 & 0 & 202 & 2 & 210 \\
\hline & $\begin{array}{l}\% \\
\text { Nationa } \\
\text { lity }\end{array}$ & $2.9 \%$ & $0.0 \%$ & $0.0 \%$ & $0.0 \%$ & $96.2 \%$ & $1.0 \%$ & $100.0 \%$ \\
\hline & $\begin{array}{l}\% \\
\text { Insuran } \\
\text { ce }\end{array}$ & $.3 \%$ & $0.0 \%$ & $0.0 \%$ & $0.0 \%$ & $2.1 \%$ & $.2 \%$ & $1.5 \%$ \\
\hline \multirow{3}{*}{$\begin{array}{l}\text { Romani } \\
\text { a }\end{array}$} & Count & 21 & 9 & 18 & 6 & 44 & 16 & 114 \\
\hline & $\begin{array}{l}\% \\
\text { Nationa } \\
\text { lity }\end{array}$ & $18.4 \%$ & $7.9 \%$ & $15.8 \%$ & $5.3 \%$ & $38.6 \%$ & $\begin{array}{c}14.0 \\
\%\end{array}$ & $100.0 \%$ \\
\hline & $\begin{array}{l}\% \\
\text { Insuran } \\
\text { ce }\end{array}$ & $1.1 \%$ & $1.4 \%$ & $2.5 \%$ & $4.9 \%$ & $.5 \%$ & $1.4 \%$ & $.8 \%$ \\
\hline \multirow[t]{3}{*}{ Russia } & Count & 167 & 2 & 217 & 4 & 704 & 51 & 1145 \\
\hline & $\begin{array}{l}\% \\
\text { Nationa } \\
\text { lity }\end{array}$ & $14.6 \%$ & $.2 \%$ & $19.0 \%$ & $.3 \%$ & $61.5 \%$ & $4.5 \%$ & $100.0 \%$ \\
\hline & $\begin{array}{l}\text { \% } \\
\text { Insuran } \\
\text { ce }\end{array}$ & $8.8 \%$ & $.3 \%$ & $30.3 \%$ & $3.3 \%$ & $7.4 \%$ & $4.5 \%$ & $8.2 \%$ \\
\hline \multirow[t]{3}{*}{ Serbia } & Count & 24 & 6 & 0 & 29 & 50 & 15 & 124 \\
\hline & $\begin{array}{l}\% \\
\text { Nationa } \\
\text { lity }\end{array}$ & $19.4 \%$ & $4.8 \%$ & $0.0 \%$ & $23.4 \%$ & $40.3 \%$ & $\begin{array}{c}12.1 \\
\%\end{array}$ & $100.0 \%$ \\
\hline & $\begin{array}{l}\% \\
\text { Insuran } \\
\text { ce }\end{array}$ & $1.3 \%$ & $.9 \%$ & $0.0 \%$ & $23.6 \%$ & $.5 \%$ & $1.3 \%$ & $.9 \%$ \\
\hline Turkey & Count & 61 & 8 & 6 & 5 & 44 & 22 & 146 \\
\hline
\end{tabular}


Healthcare Resources Consumed by Foreign Patients in the Public Hospitals of east Macedonia and Thrace

\begin{tabular}{|c|c|c|c|c|c|c|c|c|}
\hline & $\begin{array}{l}\% \\
\text { Nationa } \\
\text { lity }\end{array}$ & $41.8 \%$ & $5.5 \%$ & $4.1 \%$ & $3.4 \%$ & $30.1 \%$ & $\begin{array}{c}15.1 \\
\%\end{array}$ & $100.0 \%$ \\
\hline & $\begin{array}{l}\% \\
\text { Insuran } \\
\text { ce }\end{array}$ & $3.2 \%$ & $1.2 \%$ & $.8 \%$ & $4.1 \%$ & $.5 \%$ & $1.9 \%$ & $1.0 \%$ \\
\hline \multirow[t]{3}{*}{ Ukraine } & Count & 35 & 4 & 3 & 0 & 115 & 3 & 160 \\
\hline & $\begin{array}{l}\% \\
\text { Nationa } \\
\text { lity }\end{array}$ & $21.9 \%$ & $2.5 \%$ & $1.9 \%$ & $0.0 \%$ & $71.9 \%$ & $1.9 \%$ & $100.0 \%$ \\
\hline & $\begin{array}{l}\% \\
\text { Insuran } \\
\text { ce }\end{array}$ & $1.8 \%$ & $.6 \%$ & $.4 \%$ & $0.0 \%$ & $1.2 \%$ & $.3 \%$ & $1.1 \%$ \\
\hline \multirow{3}{*}{$\begin{array}{l}\text { United } \\
\text { Kingdo } \\
\mathrm{m}\end{array}$} & Count & 20 & 60 & 7 & 4 & 71 & 21 & 183 \\
\hline & $\begin{array}{l}\% \\
\text { Nationa } \\
\text { lity }\end{array}$ & $10.9 \%$ & $32.8 \%$ & $3.8 \%$ & $2.2 \%$ & $38.8 \%$ & $\begin{array}{c}11.5 \\
\%\end{array}$ & $100.0 \%$ \\
\hline & $\begin{array}{l}\% \\
\text { Insuran } \\
\text { ce }\end{array}$ & $1.1 \%$ & $9.0 \%$ & $1.0 \%$ & $3.3 \%$ & $.7 \%$ & $1.8 \%$ & $1.3 \%$ \\
\hline
\end{tabular}

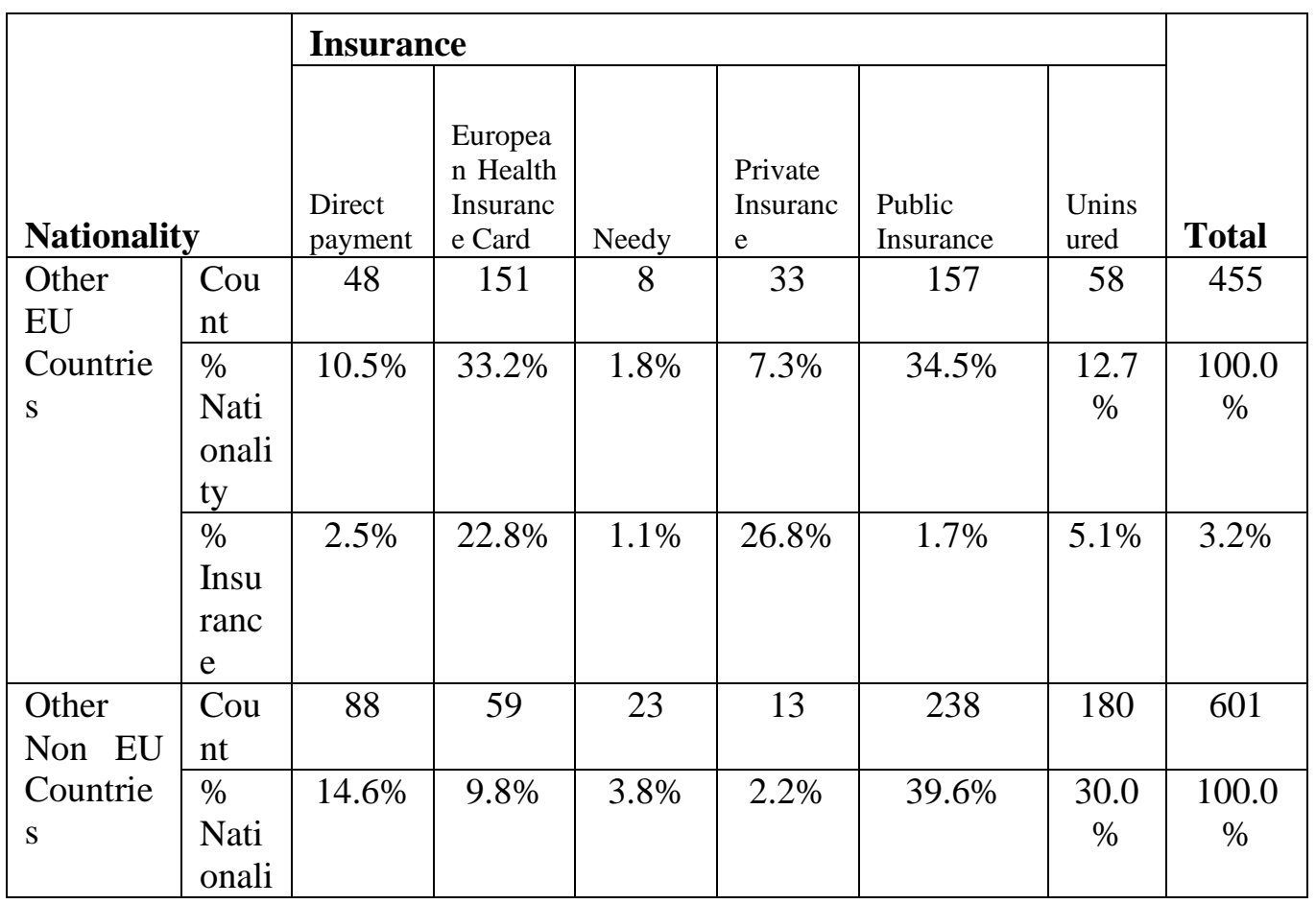


International Journal of Economics \& Business Administration, I(2)2014

134 C. Tsitsakis - C. Batzios - G. Florou - A. Karasavvoglou - P. Polychronidou

\begin{tabular}{|c|c|c|c|c|c|c|c|c|}
\hline & ty & & & & & & & \\
\hline & $\begin{array}{l}\% \\
\text { Insu } \\
\text { ranc } \\
\mathrm{e}\end{array}$ & $4.6 \%$ & $8.9 \%$ & $3.2 \%$ & $10.6 \%$ & $2.5 \%$ & $\begin{array}{l}15.7 \\
\%\end{array}$ & $4.3 \%$ \\
\hline \multirow[t]{3}{*}{ Total } & $\begin{array}{l}\text { Cou } \\
\text { nt }\end{array}$ & 1,903 & 663 & 717 & 123 & 9,482 & $\begin{array}{l}1,14 \\
6\end{array}$ & 14,034 \\
\hline & $\begin{array}{l}\% \\
\text { Nati } \\
\text { onali } \\
\text { ty }\end{array}$ & $13.6 \%$ & $4.7 \%$ & $5.1 \%$ & $.9 \%$ & $67.6 \%$ & $8.2 \%$ & $\begin{array}{l}100.0 \\
\%\end{array}$ \\
\hline & $\begin{array}{l}\% \\
\text { with } \\
\text { in } \\
\text { Insu } \\
\text { ranc } \\
\text { e }\end{array}$ & $\begin{array}{l}100.0 \\
\%\end{array}$ & $\begin{array}{l}100.0 \\
\%\end{array}$ & $100 \%$ & $\begin{array}{l}100.0 \\
\%\end{array}$ & $100.0 \%$ & $\begin{array}{l}100 . \\
0 \%\end{array}$ & $\begin{array}{l}100.0 \\
\%\end{array}$ \\
\hline
\end{tabular}

From the data above, we can see that $67.6 \%$ of foreign patients, who were treated, were covered by a public insurance organization, $13.6 \%$ paid directly for the hospital services they received, $4,7 \%$ owned a European health insurance card, $5.1 \%$ were characterized as needy, $0,9 \%$ were covered by a private organization insurance, and $8.2 \%$ were uninsured. Of the $8.2 \%$ who were uninsured, $30.1 \%$ were from Albania, $20.5 \%$ from Bulgaria, $7.6 \%$ from Iraq, $15.7 \%$ for the other non EU countries, while the rest were distributed among the other nationalities.

As far as the frequency per clinic of inpatient care is concerned, the data from table 5 (see below) shows that for Kavala, Didimoticho and Xanthi, 26.4\% of the foreign patients were females who needed the obstetric-gynecology clinic services, in most cases to give birth, $13.2 \%$ needed surgery (which in most cases was necessary to treat acute health problems), $11.3 \%$ were children who received healthcare services, $8.8 \%$ were treated in the pathology clinic, $9.4 \%$ received one day treatment in the one day pathology and psychiatry clinic, while the rest of them were distributed among the other clinics. 
Table 5. Foreign Patients in the Region of Eastern Macedonia and Thrace According to the Clinic Where They Were Treated for the Years 2005-2011

\begin{tabular}{|l|l|l|l|}
\hline Clinic & Frequency & $\begin{array}{l}\text { Perce } \\
\text { nt }\end{array}$ & $\begin{array}{l}\text { Cumulative } \\
\text { Percent }\end{array}$ \\
\hline Obstetric-Gynecology & 3,290 & 26.4 & 26.4 \\
\hline Surgery & 1,640 & 13.2 & 39.6 \\
\hline Pediatric & 1,404 & 11.3 & 50.9 \\
\hline Pathology & 1,101 & 8.8 & 59.7 \\
\hline One day & 974 & 7.8 & 67.6 \\
\hline Orthopedic & 725 & 5.8 & 73.4 \\
\hline Premature & 558 & 4.5 & 77.9 \\
\hline Lung & 457 & 3.7 & 81.5 \\
\hline Urology & 434 & 3.5 & 85.0 \\
\hline Cardiology & 354 & 2.8 & 87.9 \\
\hline
\end{tabular}

\begin{tabular}{|c|c|c|c|}
\hline Clinic & Frequency & Percent & $\begin{array}{c}\text { Cumulative } \\
\text { Percent }\end{array}$ \\
\hline Ear Nose and Throat & 282 & 2.3 & 90.1 \\
\hline Neurology & 247 & 2.0 & 92.1 \\
\hline One day Psychiatry & 196 & 1.6 & 93.7 \\
\hline Rheumatology & 153 & 1.2 & 94.9 \\
\hline Ophthalmology & 130 & 1.0 & 96.0 \\
\hline Neurosurgery & 126 & 1.0 & 97.0 \\
\hline Psychiatry & 120 & 1.0 & 97.9 \\
\hline Nephrology & 110 & .9 & 98.8 \\
\hline Thalassaemia & 45 & .4 & 99.2 \\
\hline Oncology & 43 & .3 & 99.5 \\
\hline Intensive Care Unit & 25 & .2 & 99.7 \\
\hline Phototherapy & 22 & .2 & 99.9 \\
\hline Incubator & 12 & .1 & 100.0 \\
\hline
\end{tabular}

As far as concerning the loss of the hospitals from unreceived collectibles, we performed a bivariate analysis to find the frequencies and calculate percents which 
allow us to make comparisons between the ethnicity group of patients and their insurance status.

As shown in Table 6, over the period 2005-2011 about $97 \%$ of the inpatients were Greek citizens, while the remaining 3\% were foreign patients (about $0.5 \%$ from EU countries and the rest $2.5 \%$ from non EU countries). The group of the foreign patients from non EU countries, especially the part of the group that is uninsured is the group that includes the undocumented immigrants. However, the uninsured part of this group may also include documented immigrants, who were unemployed for a period of over 12 months and were no longer covered by a public insurance fund. From table 6, we can see that this group accounts for only about $0.25 \%$ of the inpatients.

Table 6: Bivariate Analysis Insurance*Nationality for the Public Hospitals of Eastern Macedonia and Thrace Region from 2005 to 2007

\begin{tabular}{|c|c|c|c|c|c|c|c|}
\hline & \multirow{2}{*}{\multicolumn{3}{|c|}{ Insurance }} & \multicolumn{3}{|c|}{ Nationality } & \multirow[b]{2}{*}{ Total } \\
\hline & & & & $\begin{array}{l}\text { EU } \\
\text { Countries }\end{array}$ & Greece & $\begin{array}{l}\text { Non EU } \\
\text { Countries }\end{array}$ & \\
\hline \multirow{8}{*}{2005} & \multirow[t]{6}{*}{ Insurance } & \multirow{2}{*}{$\begin{array}{l}\text { Private } \\
\text { Insurance }\end{array}$} & Count & 33 & 1,091 & 227 & 1,351 \\
\hline & & & $\%$ of Total & $.05 \%$ & $1.71 \%$ & $.36 \%$ & $2.12 \%$ \\
\hline & & \multirow{2}{*}{$\begin{array}{l}\text { Public } \\
\text { Insurance }\end{array}$} & Count & 103 & 58,738 & 878 & 59,719 \\
\hline & & & $\%$ of Total & $.16 \%$ & $92.24 \%$ & $1.38 \%$ & $93.78 \%$ \\
\hline & & \multirow[t]{2}{*}{ Uninsured } & Count & 28 & 2,361 & 223 & 2,612 \\
\hline & & & $\%$ of Total & $.04 \%$ & $3.71 \%$ & $.35 \%$ & $4.10 \%$ \\
\hline & \multirow{2}{*}{\multicolumn{2}{|c|}{ Total }} & Count & 164 & 62,190 & 1.328 & 63,682 \\
\hline & & & $\%$ of Total & $.26 \%$ & $97.66 \%$ & $2.09 \%$ & $100.00 \%$ \\
\hline \multirow{8}{*}{2006} & \multirow[t]{6}{*}{ Insurance } & \multirow{2}{*}{$\begin{array}{l}\text { Private } \\
\text { Insurance }\end{array}$} & Count & 31 & 1.111 & 212 & 1.354 \\
\hline & & & $\%$ of Total & $.05 \%$ & $1.75 \%$ & $.33 \%$ & $2.1 \%$ \\
\hline & & \multirow{2}{*}{$\begin{array}{l}\text { Public } \\
\text { Insurance }\end{array}$} & Count & 76 & 58,193 & 1,124 & 59,393 \\
\hline & & & $\%$ of Total & $.12 \%$ & $91.77 \%$ & $1.77 \%$ & $93.7 \%$ \\
\hline & & \multirow[t]{2}{*}{ Uninsured } & Count & 18 & 2,428 & 221 & 2,667 \\
\hline & & & $\%$ of Total & $.03 \%$ & $3.83 \%$ & $.35 \%$ & $4.2 \%$ \\
\hline & \multirow{2}{*}{\multicolumn{2}{|c|}{ Total }} & Count & 125 & 61,732 & 1,557 & 63,414 \\
\hline & & & $\%$ of Total & $.20 \%$ & $97.35 \%$ & $2.46 \%$ & $100.0 \%$ \\
\hline \multirow{4}{*}{2007} & \multirow[t]{4}{*}{ Insurance } & \multirow{2}{*}{$\begin{array}{l}\text { Private } \\
\text { Insurance }\end{array}$} & Count & 70 & 1,100 & 174 & 1,344 \\
\hline & & & $\%$ of Total & $.11 \%$ & $1.76 \%$ & $.28 \%$ & $2.16 \%$ \\
\hline & & \multirow{2}{*}{$\begin{array}{l}\text { Public } \\
\text { Insurance }\end{array}$} & Count & 279 & 57,159 & 1,037 & 58,475 \\
\hline & & & $\%$ of Total & $.45 \%$ & $91.66 \%$ & $1.66 \%$ & $93.77 \%$ \\
\hline
\end{tabular}


Healthcare Resources Consumed by Foreign Patients in the Public Hospitals of east Macedonia and Thrace

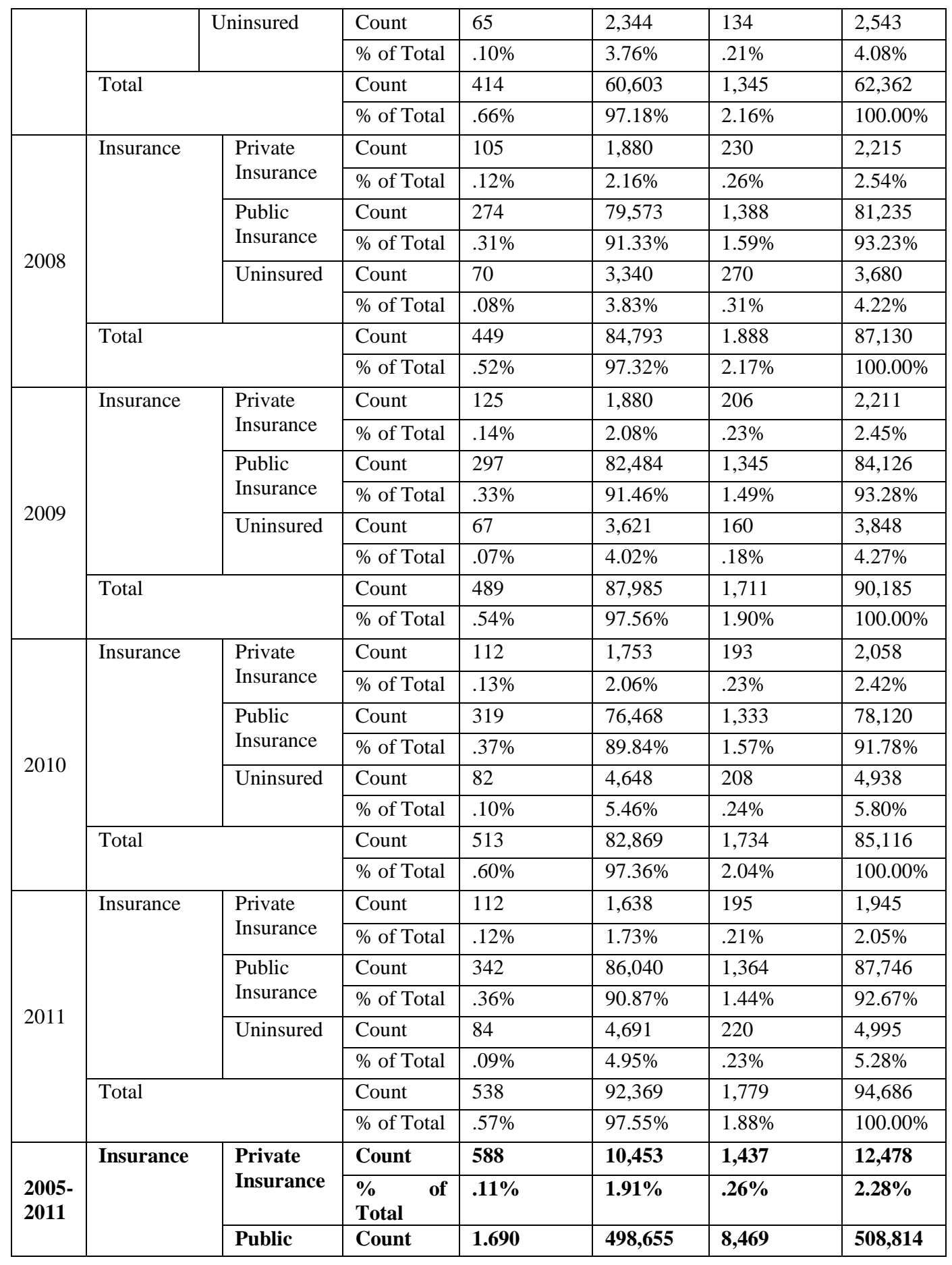


International Journal of Economics \& Business Administration, I(2)2014

138 C. Tsitsakis - C. Batzios - G. Florou - A. Karasavvoglou - P. Polychronidou

\begin{tabular}{|c|c|c|c|c|c|c|}
\hline & Insurance & $\begin{array}{ll}\% & \text { of } \\
\text { Total }\end{array}$ & $.31 \%$ & $91.23 \%$ & $1.55 \%$ & $93.09 \%$ \\
\hline & Uninsured & Count & 414 & 23,433 & 1,436 & 25,283 \\
\hline & & $\begin{array}{ll}\% & \text { of } \\
\text { Total }\end{array}$ & $.08 \%$ & $4.29 \%$ & $.26 \%$ & $4.63 \%$ \\
\hline \multirow{2}{*}{\multicolumn{2}{|c|}{ Total }} & Count & 2.692 & 532,541 & 11,342 & 546,575 \\
\hline & & $\begin{array}{ll}\% & \text { of } \\
\text { Total }\end{array}$ & $.49 \%$ & $97.43 \%$ & $2.08 \%$ & $100.00 \%$ \\
\hline
\end{tabular}

Table 7 below presents the relationship between the total revenue (pricing) of the hospitals and the amount that represent the loss of the hospitals from uncollectible receivables relating to foreign inpatients. As we can see, this amount accounts for about $0.5 \%$ of the total revenues (about $0.05 \%$ from uninsured patients from EU countries and the remaining $0.45 \%$ from uninsured patients from non EU countries). It must be said that this percentage is very low compared to the total hospital revenue $(273,287,123 €)$.

Table 7: Total Revenue of the Hospitals vs Uncollectible Revenues from Healthcare Services Provided to Uninsured Foreign Patients

\begin{tabular}{|l|l|l|l|l|l|}
\hline Year & Total pricing & $\begin{array}{l}\text { EU countries } \\
\text { Uninsured }\end{array}$ & $\%$ Total & $\begin{array}{l}\text { Non } \\
\text { countries } \\
\text { Uninsured }\end{array}$ & \% Total \\
\hline 2005 & $27,340,274.02$ & $4,649.55$ & $0.02 \%$ & $138,807.85$ & $0.51 \%$ \\
\hline 2006 & $29,006,236.44$ & $2,972.70$ & $0.01 \%$ & $195,561.61$ & $0.67 \%$ \\
\hline 2007 & $31,926,845.25$ & $31,111.57$ & $0.10 \%$ & $428,381.71$ & $1.34 \%$ \\
\hline 2008 & $47,898,237.75$ & $35,063.67$ & $0.07 \%$ & $130,122.78$ & $0.27 \%$ \\
\hline 2009 & $49,488,804.20$ & $25,796.10$ & $0.05 \%$ & $97,779.53$ & $0.20 \%$ \\
\hline 2010 & $41,658,168.73$ & $27,111.46$ & $0.07 \%$ & $153,928.72$ & $0.37 \%$ \\
\hline 2011 & $45,968,556.88$ & $24,596.03$ & $0.05 \%$ & $109,920.98$ & $0.24 \%$ \\
\hline $2005-$ & $273.287 .123,27$ & $151.301,08$ & $0.06 \%$ & 1.254 .503 .18 & $0.46 \%$ \\
\hline 2011 & & & & & \\
\hline
\end{tabular}

\section{Discussion}

The change in epidemiologic standards, the rapid development of medical science and the new state of art medical technology has led to new treatments for various health problems. Previously fatal diseases have become chronic and life expectancy has risen significantly, causing healthcare costs to explode. As a result, rising healthcare expenditures is a reality that all developed countries have to deal with.

On the other hand, health spending in Greece decreased by 11\%, in both 2010 and 2011, due to the economic crisis, while the same crisis caused an increased demand for public health services, since access to private sector health services is impossible 
for most of the Greek citizens. As the crisis deepens, the gap between the demand and offer of public healthcare services is becoming wider and phenomena such as xenophobia and racism are becoming more intense. Populist politicians find their scapegoats in the immigrant community, ascribing the chronic problems of the Greek economy and society to them and not to its structural problems and corruption. Newspapers and internet "information" make this systematic scapegoating more intense, referring to jobs lost because of immigrants and hospital beds occupied for free by foreigners, while Greek citizens die as a result of not having the money needed to pay the hospitals.

However, the reality may be different from these populistic cries. Lianos (2003) claims that the unemployment rate of native workers is not affected by the presence of immigrants, while Bagavos and Papadopoulou (2006) argue that the entry of immigrants has postponed the ageing population problem and contributed to the survival of the insurance system.

Our study, based on data from the public hospitals of Eastern Macedonia and Thrace for the period 2005-2011, has proven that in this region at least, the foreign patients, especially those undocumented, use disproportionately fewer medical services and contribute less to health care costs in relation to their population share. This finding matches those of other similar studies for other countries (Goldman et al., 2006) and probably can be explained either by the immigrants' better health because of their younger age or by their lack of health insurance.

However, our study is limited to the region of Eastern Macedonia and Thrace, where there is not a heavy concentration of immigrants. Healthcare costs could be large in regions where they are heavily concentrated (e.g. Athens). Research on immigration healthcare service consumption is limited by severe constraints on the quantity and quality of available data. In this general political and economic context, it will be crucial to counteract xenophobia and racism and not to retreat from efforts to establish and implement national migrant health policies.

The immigrant population in Greece is quite considerable and may be more considerable in the future and healthcare costs are only one of the major components around which a policy debate about the fiscal benefits or burden of immigrants should focus. A comprehensive analysis should incorporate the taxes paid by immigrants, and all the public benefits they received, not only health care.

Although there is an increasing harmonization of immigration policies in EU member countries, the dominant emphasis has been on restriction and control (Bendel 2007). However, restrictions in health care provision to undocumented immigrants, do not solve the problem of immigration, they rather operate as blankets hiding it. The hidden problems could become bigger and threaten the public health. 
In order to be able to design and apply an effective immigrant healthcare policy, we need accurate and homogenous data, about the foreign patients treated in Greek hospitals, which should help to establish a climate that can help the health system to become more responsive and sensitive to the needs of immigrants (Ingleby 2006, Rechel et al., 2011).

\section{Acknowledgements}

This research was supported by the Project "Immigrants and Health Services - The case of Eastern Macedonia and Thrace region" that is co-funded by the European Union (European Social Fund) and National Resources - ARCHIMEDES III.

\section{References}

Bagavos, Ch. and Papadopoulou, D., 2006. Immigration and Immigrants' Incorporation to the Greek Society. Scientific Company of Social Policy, 2006 (in Greek).

Baldwin-Edwards, M., 2004. Immigration into Greece, 1990-2003: A Southern European Paradigm? Paper presented at the European Population Forum 2004, Mediterranean Migration Observatory.

Bendel, P., 2007. Everything under control? The European Union's policies and politics of immigration. In: Faist, T. and Ette, A. (eds) The Europeanization of National Policies and Politics of Immigration. Basingstoke: Palgrave Macmillan: 32-48.

E72454.pdf (2014-05-15).

Economou, C., 2010. Greece: Health system review, Health Systems in Transition, 12 (7):1180.

Ingleby., D., 2006. Getting multicultural health care off the ground: Britain and the

Kentikelenis, A., Karanikolos, M., Papanicolas, I., et al., 2011. Health effects of financial crisis: omens of a Greek tragedy. Lancet, 378:1457-8.

Kentikelenis, A., Papanicolas, I., 2012. Economic crisis, austerity and the Greek public health system. European journal of public health, 22(1):4-5.

Maroufof, M,. 2013. Irregular migration between Georgia and Greece. Everyone can cross a low fence. Deliverable 2.1. Background Report: Migration System 2 (Georgia). Athens: IRMA, 2013. p. 18-19.

Médecins du Monde, 2009. Access to Healthcare for Undocumented. European Observatory on Access to Healthcare.

Netherlands compared. International Journal of Migration, Health and Social Care,

OECD, 2012. Health Data 2012. June 2012 edition.

Rechel, B., Mladovsky, P., Devillé, W., et al., 2011. Migration and health in the European Union: an introduction. In: Rechel B, Mladovsky P, Devillé W et al, eds.Migration and health in the European Union. Maidenhead: Open University Press.

Tragakes, E. and Polyzos, N., 1996. Health Systems in Transition: Greece. Regional Office for Europe.http://www.euro.who.int/_data/assets/pdf_file/0020/120278/

Vidali, M., 1999. Immigration: Living in a policy vacuum. The plight of Albanian immigrants in Greece, Central Europe Review, Vol 1, No 21, 15. 